\title{
Research on Settlement Prediction of Small Water Conservancy Project based on ELM Model Optimized by Genetic Algorithm
}

\author{
Ruizi Yang ${ }^{1, *}$ \\ ${ }^{1}$ Management Science and Engineering, School of management, Tianjin University of Technology, China
}

\begin{abstract}
To find suitable for small water conservancy engineering standard method for prediction of subsidence. This paper based on the genetic algorithm GA optimization extreme learning machine, three different ELM model activation function. From this, six computational models are obtained. According to the input of groundwater dynamic changes, precipitation, temperature and soil four indicators of the two kinds of input combinations, a total of 12 kinds of model input. It's concluded that the optimal settlement prediction

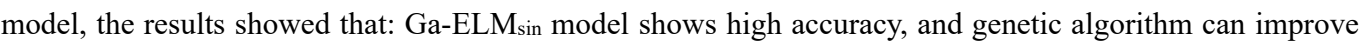
the calculation accuracy of ELM model. Groundwater dynamics is the main factor affecting settlement.
\end{abstract}

\section{Introduction}

With the growth of the domestic population and the rapid development of the economy, the requirements for the quality of the project are also gradually improved in the limited land resources. It is the focus of relevant industries to promote engineering development and improve residents' quality of life $\mathrm{e}^{[1-2]}$. Small water conservancy projects are closely related to the life of residents. China's water conservancy industry is mainly concentrated in the construction of small water conservancy projects, which have direct and obvious benefits and are directly related to residents' daily life and water use for crops, and are of great significance to the improvement of residents' life ${ }^{[3-4]}$. The quality and operation management of small water conservancy projects may directly affect the daily work and life of residents. However, due to the small water conservancy projects' less investment and remote location, the implementation degree of project quality monitoring is low, which seriously affects their service life.

Settlement is one of the main reasons that affect the service life and durability of a project. The settlement is mainly affected by natural factors and human factors, resulting in deformation in the area where the project is located and resulting in certain damage to the main structure of the project due to additional stress ${ }^{[5]}$.Longterm settlement observation shows that there is a certain distribution law in the process from the construction period to the stable period, but the settlement is not easy to be discovered and has a long periodicity, which has become an important geological disaster and has been listed as the focus of prevention and observation ${ }^{[6]}$.

With the increasingly serious influence of settlement, it has become the research of relevant departments to find out the reasonable settlement prediction method while observing the settlement daily. Among them, the machine learning neural network model is widely used. Wang $\mathrm{Lu}$ and Gui Zhanfei ${ }^{[7]}$ established a building settlement prediction model based on GM-ARMA-BP model, and pointed out that this combined model overcame the disadvantages of the single model. By integrating the advantages of each model, the prediction accuracy of the comprehensive model was improved by at least $50 \%$ compared with that of the single model. Feng Shaoquan et al. ${ }^{[8]}$ established a prediction model for the settlement amount of elevated bridge piers based on the combined model of $G A-B P-M C$, and proved the scientific nature of the model based on the index of absolute error and relative error. Song Chuping ${ }^{[9]}$ also established the settlement prediction model of deep foundation pit based on the improved BP neural network model, and pointed out that the improved BP neural network model had faster convergence speed and higher generalization ability. Peng Yuan ${ }^{[10]}$ used genetic algorithm to optimize BP neural network model to predict the settlement of buildings, and achieved good results.

Extreme learning machine (ELM) is a new neural network algorithm. This algorithm overcomes the shortcoming of slow convergence speed of traditional BP neural network model and is applied to data training and prediction. The genetic algorithm facilitates the model to find the optimal path and find out the rules of training data. In this paper, a settlement prediction model for small water conservancy projects is established based on the ultimate learning machine (GA-ELM) algorithm of genetic optimization.

\section{Research methods}

\subsection{Extreme learning machine model (ELM)}

Ultimate Learning machine model (ELM) is a singlehidden layer feedforward algorithm model, which is

\footnotetext{
" Corresponding author: ruiz193144302@stud.tjut.edu.cn
} 
mainly composed of input layer, hidden layer and output layer. Its basic structure is shown in Figure 1.

Let the activation function of hidden layer neurons be $g(\omega, X, b)$, then the expression of output layer of ELM model is:

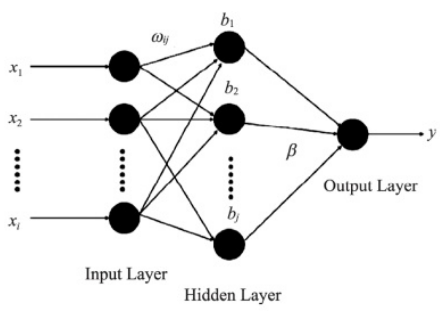

Fig. 1. ELM model basic structure.

$$
F\left(X_{i}\right)=\sum_{j=1}^{m} \beta_{j} g\left(\omega_{i j} X_{i}+b_{j}\right)
$$

Where $\beta_{j}$ - the connection value between the hidden layer and the output layer; $\omega_{i j}$ - the connection value between the input layer and the hidden layer; $X_{i}$ - Input layer variable value; $b_{j}$ - threshold for the JTH hidden layer.

The activation function of ELM model can be divided into three types: Sine function, Radbas function and Hardlim function. In this paper, three activation functions are respectively used to establish the settlement prediction model for small water conservancy projects. The specific formulas of the three functions are as follows:

Sine function $E L M_{\text {sin }}$ :

$$
g\left(\omega_{i j}, X_{i}, b_{j}\right)=g\left(\omega_{i j}, X_{i}+b_{j}\right)=\sin \left(\omega_{i j}, X_{i}+b_{j}\right)
$$

Radbas function $E L M_{\text {rad }}$ :

$$
g\left(\omega_{i j}, X_{i}, b_{j}\right)=\exp \left(-\frac{\left\|X-\omega_{i j}\right\|^{2}}{b_{j}^{2}}\right)
$$

Hardlim function $E L M_{\text {hard }}$ :

$$
g\left(\omega_{i j}, X_{i}, b_{j}\right)= \begin{cases}1, & \omega_{i j} X+b_{j} \geq 0 \\ 2, & \omega_{i j} X+b_{j}<0\end{cases}
$$

\subsection{Genetic algorithm optimization of extreme learning machine model (GA-ELM)}

The application of the principle of genetic algorithm in the optimization of neural network learning can help the neural network model find the optimal solution as soon as possible and shorten the training time of the model. The specific steps for ELM model optimization are as follows: determine the ELM calculation structure and determine the length of the model calculation based on the original number of settlement data; Based on genetic algorithm, the fitness value of each subsidence data individual is calculated to find the optimal solution. Finally, the $G A$ ELM model is determined and the predicted data is obtained. The specific steps are shown in Figure 2.

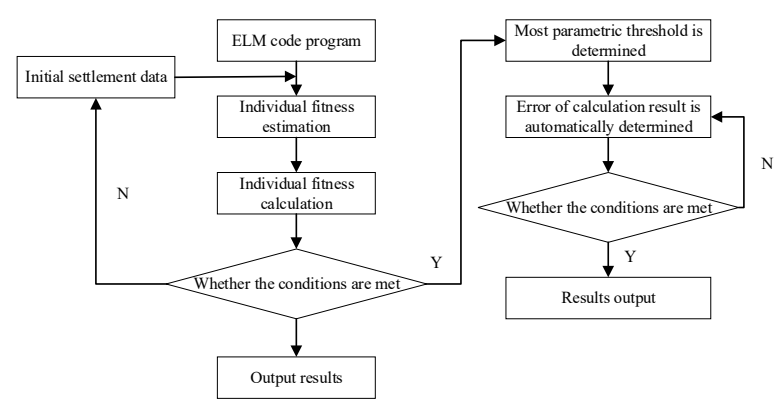

Fig.2. Ga-ELM model calculation steps.

\subsection{Model training and validation}

The subsidence of a small water conservancy project is mainly related to the groundwater dynamics, precipitation, air temperature and soil quality changes of the project site. The dynamic of groundwater is the main factor that affects the settlement of projects. Three activation function of the genetic algorithm to optimize the ELM model, based on four kinds of influence factors of 2 kinds of combinations: 1 enter groundwater dynamic combination, precipitation, temperature and soil changes in four indicators, combination of two input precipitation, temperature and soil changes in three kinds of index, specific combination forms are shown in table 1, we validate the calculation

\begin{tabular}{|c|c|c|}
\hline Model & Input parameters & Model code \\
\hline 1. GA-ELM ${ }_{\sin }$ & \multirow{6}{*}{$\begin{array}{l}\text { Groundwater dynamics, } \\
\text { precipitation, } \\
\text { air temperature } \\
\text { and soil quality }\end{array}$} & ELM $_{1}$ \\
\hline 2. GA-ELM ${ }_{\mathrm{rad}}$ & & $\mathrm{ELM}_{2}$ \\
\hline 3. GA-ELM ${ }_{\text {hard }}$ & & $\mathrm{ELM}_{3}$ \\
\hline 4.ELM $\mathrm{E}_{\sin }$ & & $\mathrm{ELM}_{4}$ \\
\hline 5. ELM rad & & $\mathrm{ELM}_{5}$ \\
\hline 6. ELM hard $_{\text {h }}$ & & $\mathrm{ELM}_{6}$ \\
\hline 7. GA-ELM ${ }_{\sin }$ & \multirow{6}{*}{$\begin{array}{l}\text { Changes in precipitation, } \\
\text { air temperature } \\
\text { and soil quality }\end{array}$} & $\mathrm{ELM}_{7}$ \\
\hline 8. GA-ELM ${ }_{\mathrm{rad}}$ & & $\mathrm{ELM}_{8}$ \\
\hline 9. GA-ELM ${ }_{\text {hard }}$ & & $\mathrm{ELM}_{9}$ \\
\hline 10.ELM sin $_{\text {sin }}$ & & ELM $_{10}$ \\
\hline 11. ELMrad & & ELM $_{11}$ \\
\hline 12. ELM hard & & ELM $_{12}$ \\
\hline
\end{tabular}
precision of different models under different input methods.

Table 1. Model parameter input.

\subsection{Model accuracy verification}

Take root mean square error (RMSE), relative root mean square error (RRMSE), determination coefficient $\left(R^{2}\right)$, model efficiency coefficient $\left(E_{n s}\right)$ and average absolute error $(M A E)$ as the model accuracy evaluation index system, and the specific formula is as follows:

$$
\begin{gathered}
R M S E=\sqrt{\frac{1}{m} \sum_{i=1}^{m}\left(Y_{i}-X_{i}\right)^{2} \times 100 \%} \\
R R M S E=\frac{\sqrt{\frac{1}{m} \sum_{i=1}^{m}\left(Y_{i}-X_{i}\right)^{2}}}{\bar{X}} \times 100 \% \\
R^{2}=\frac{\left[\sum_{i=1}^{m}\left(X_{i}-\bar{X}\right)\left(Y_{i}-\bar{Y}\right)\right]^{2}}{\sum_{i=1}^{m}\left(X_{i}-\bar{X}\right)^{2} \sum_{i=1}^{m}\left(Y_{i}-\bar{y}\right)^{2}} \\
M A E=\frac{1}{m} \sum_{i=1}^{m}\left|Y_{i}-X_{i}\right|
\end{gathered}
$$




$$
E_{n s}=1-\frac{\sum_{i=1}^{m}\left(Y_{i}-X_{i}\right)^{2}}{\sum_{i=1}^{m}\left(X_{i}-\bar{X}\right)^{2}}
$$

Where, $Y_{i}$ - different methods to process values; $X_{i}$ - the measured standard value; The average of $\bar{X}-X_{i} ; m$ Number of data samples.

$G P I$ index is introduced to comprehensively judge the accuracy of different models. The specific formula is as follows:

$$
G P I=\sum_{j=1}^{5} \alpha_{j}\left(g_{j}-y_{i j}\right)
$$

Where, $\alpha_{j}$ - constant; $M A E$ and RRMSE are $1, N S$ is $1 ; g_{j}$ - the median of the scaling values of different indicators; $Y_{i j}$ - the scale values of different indicators.

\section{Results and analysis}

\subsection{Comparison of settlement fitting effect between different models}

Comparison and analysis of settlement prediction results and measured values by different models are shown in Figure 3. As can be seen from Figure 3, the simulation accuracy of different prediction models is different, but the overall data change trend is basically consistent with the measured value change trend. The settlement value of different parts of the building is different, the settlement value of the edge of the building is higher than that of the inside of the building. The $E L M_{1}$ model performs well in the prediction of building edge and internal settlement, with the simulated value closest to the measured value, while the $E L M_{12}$ model has the worst fitting effect.

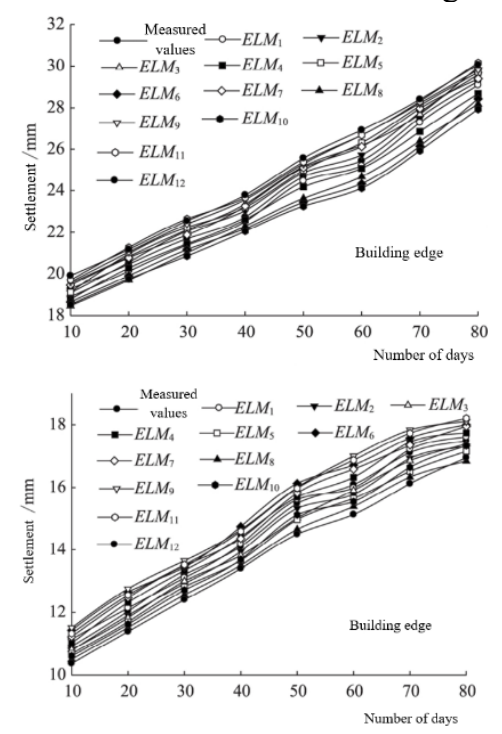

Fig. 3. Comparison of fitting effect between different model settlement prediction and measured value

\subsection{Comparison of settlement prediction accuracy of different models}

The accuracy of the prediction results of different models is shown in Table 2. As can be seen from Table 2, there are certain differences in the accuracy indexes of different models, and the accuracy of models at different positions is different. At the edge of the building, The Precision of $E L M_{1}$ model is the highest, and the values of $R M S E$, RRMSE, $R^{2}, E N S$ and $M A E$ are $1.719 \mathrm{~mm}, 12.7 \%, 0.964$, 0.946 and $1.283 \mathrm{~mm}$ respectively. The model has the lowest error and the highest consistency with the measured value, while the precision of $E L M_{12}$ model is the lowest. The values of RMSE, RRMSE, $R^{2}, E_{N S}$ and $M A E$ were $3.398 \mathrm{~mm}, 25.0 \%, 0.803,0.789$ and $2.524 \mathrm{~mm}$, respectively, with the highest error and poor consistency.

Inside the building, the $E L M_{1}$ model also has the highest precision, while the $E L M_{12}$ model has the lowest precision. The values of RMSE, RRMSE, $R^{2}, E_{n S}$ and $M A E$ can be divided into 1.245 and $3.471 \mathrm{~mm}, 9.1 \%$ and $25.5 \%$, 0.974 and $0.795,0.973$ and $0.793,0.844$ and $2.403 \mathrm{~mm}$. The accuracy of the 3 activation functions of ELM model is sine function $>$ Radbas function $>$ Hardlim function. The accuracy of the ELM function optimized by genetic algorithm is generally higher than that of the unoptimized ELM model under the same activation function. At the same time, when the dynamic influence factors of groundwater are taken into account, the precision of the model can be significantly improved, RMSE can be reduced by at least $15.6 \%, R R M S E$ by at least $20.4 \%$, R2 by at least $22.6 \%, E N S$ by at least $18.7 \%$ and $M A E$ by at least $21.8 \%$.

\begin{tabular}{|c|c|c|c|c|c|}
\hline $\begin{array}{c}\text { Measuring } \\
\text { point }\end{array}$ & $\begin{array}{c}\text { Precision } \\
\text { index }\end{array}$ & ELM $_{1}$ & $\mathbf{E L M}_{2}$ & $\mathbf{E L M}_{3}$ & $\mathbf{E L M}_{4}$ \\
\hline \multirow{5}{*}{ Edge } & $R M S E / \mathrm{mm}$ & 1.719 & 1.797 & 2.033 & 2.149 \\
\hline & RRMSE/\% & 12.7 & 13.2 & 15.0 & 15.8 \\
\hline & $R^{2}$ & 0.964 & 0.96 & 0.948 & 0.941 \\
\hline & $E_{N S}$ & 0.946 & 0.941 & 0.925 & 0.916 \\
\hline & $M A E / \mathrm{mm}$ & 1.283 & 1.353 & 1.525 & 1.604 \\
\hline \multirow{5}{*}{ Internal } & $R M S E / \mathrm{mm}$ & 1.245 & 1.410 & 1.543 & 1.610 \\
\hline & RRMSE $/ \%$ & 9.1 & 10.4 & 11.3 & 11.8 \\
\hline & $R^{2}$ & 0.974 & 0.966 & 0.959 & 0.945 \\
\hline & $E_{N S}$ & 0.973 & 0.966 & 0.959 & 0.956 \\
\hline & $M A E / \mathrm{mm}$ & 0.844 & 0.945 & 1.072 & 1.077 \\
\hline $\begin{array}{c}\text { Measuring } \\
\text { point }\end{array}$ & $\begin{array}{c}\text { Precision } \\
\text { index }\end{array}$ & ELM $_{5}$ & $\mathbf{E L M}_{6}$ & $\mathbf{E L M}_{7}$ & $\mathbf{E L M}_{8}$ \\
\hline \multirow{5}{*}{ Edge } & $R M S E / \mathrm{mm}$ & 2.161 & 2.397 & 2.406 & 2.700 \\
\hline & RRMSE $/ \%$ & 15.9 & 18.4 & 17.7 & 19.9 \\
\hline & $R^{2}$ & 0.942 & 0.923 & 0.929 & 0.924 \\
\hline & $E_{N S}$ & 0.915 & 0.886 & 0.894 & 0.867 \\
\hline & $M A E / \mathrm{mm}$ & 1.632 & 1.869 & 1.798 & 2.108 \\
\hline \multirow{5}{*}{ Internal } & $R M S E / \mathrm{mm}$ & 1.538 & 1.830 & 1.760 & 1.869 \\
\hline & RRMSE $/ \%$ & 11.3 & 13.4 & 12.9 & 13.7 \\
\hline & $R^{2}$ & 0.96 & 0.943 & 0.947 & 0.941 \\
\hline & $E_{N S}$ & 0.956 & 0.943 & 0.947 & 0.94 \\
\hline & $M A E / \mathrm{mm}$ & 1.067 & 1.256 & 1.183 & 1.271 \\
\hline $\begin{array}{c}\text { Measuring } \\
\text { point }\end{array}$ & $\begin{array}{c}\text { Precision } \\
\text { index }\end{array}$ & ELM9 & $\begin{array}{c}\text { ELM1 }_{1} \\
0\end{array}$ & $\begin{array}{c}\text { ELM } \\
11\end{array}$ & $\begin{array}{c}\text { ELM } \\
12\end{array}$ \\
\hline \multirow{5}{*}{ Edge } & $R M S E / \mathrm{mm}$ & 2.809 & 2.9 & 3.068 & 3.398 \\
\hline & RRMSE $/ \%$ & 20.7 & 21.4 & 22.6 & 25.0 \\
\hline & $R^{2}$ & 0.914 & 0.904 & 0.842 & 0.803 \\
\hline & $E_{N S}$ & 0.856 & 0.847 & 0.828 & 0.789 \\
\hline & $M A E / \mathrm{mm}$ & 2.165 & 2.237 & 2.305 & 2.524 \\
\hline \multirow{5}{*}{ Internal } & $R M S E / \mathrm{mm}$ & 1.982 & 2.049 & 3.157 & 3.471 \\
\hline & RRMSE $/ \%$ & 14.6 & 15.1 & 23.2 & 25.5 \\
\hline & $R^{2}$ & 0.934 & 0.929 & 0.835 & 0.795 \\
\hline & $E_{N S}$ & 0.933 & 0.928 & 0.829 & 0.793 \\
\hline & $M A E / \mathrm{mm}$ & 1.377 & 1.427 & 2.211 & 2.403 \\
\hline
\end{tabular}

Table 2. Comparison of precision indexes of different models

\subsection{Comparison of GPI index of settlement prediction by different models}

Figure 4 shows the GPI index comparison of the prediction results of different models. It can be seen from 
the figure that $G P I$ is calculated from the simulation results of different models GPI is different at different locations. At the edge of the building, ELM $M_{1}$ model has the highest GPI (1.904), while ELM 12 model has the lowest accuracy ( -3.096$)$. Inside the building, ELM model has the highest accuracy, ELM 12 model has the lowest accuracy, and GPI is 1.052 and - 3.948. In summary, $G A-E L M_{\text {sin }}$ model is the model with the highest accuracy for settlement prediction of small water conservation projects.

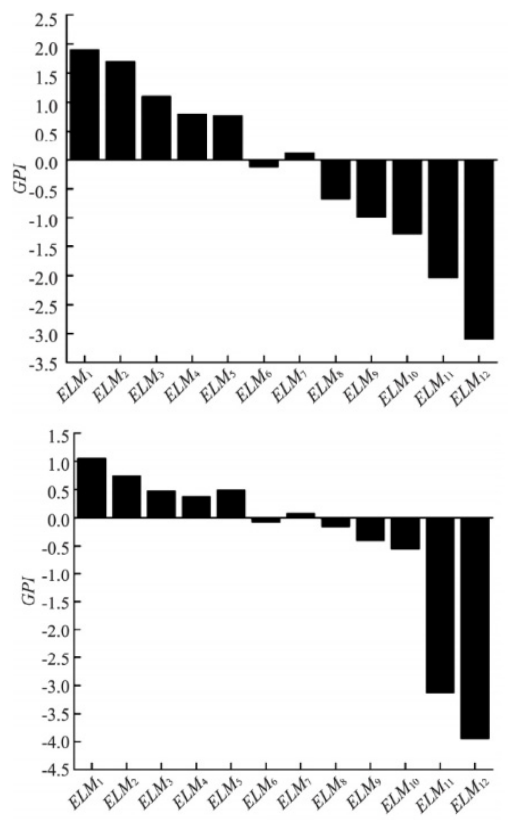

Fig. 4. Comparison of GPI index of settlement prediction by different models (the top is the edge, the bottom is the inside)

\section{Conclusion}

Based on the limit learning machine model, this paper studies the settlement prediction models of small water conservancy projects under different models. Based on the three activation functions of ELM model, genetic algorithm was used to optimize each model respectively. And the calculated value was compared with the measured value. The conclusion was drawn: although there were some differences in the accuracy of different models, the predicted value was basically consistent with the change trend of the measured value. The calculation accuracy of $E L M$ model can be improved by genetic algorithm. Among the 3 activation functions of ELM model, sine activation function has the highest accuracy. In summary, $G A-E L M_{\text {sin }}$ model can be the standard model for settlement prediction of small water conservancy projects. In the future research, the model can be compared with the generalized regression neural network model and BP neural network model to further illustrate the scientific nature of the model.

\section{References}

1. Liu Yitao. Development of BIM Technology and its Application in Water Conservancy Projects [J].Water Planning and Design, 10 (2019)
2. Zhang Weidong. Countermeasures and Suggestions for the development of Foreign Projects in China's Water Conservancy and hydropower Industry [J].Water Resources Planning and Design, 1 (2009)

3. Huangfu Mingxia, WANG Wenting, LIU Yuqing. Quality control measures for construction of small Irrigation and water conservancy Projects [J]. Henan water conservancy and south-north water diversion project, 48 ,5 (2019)

4. Lu Yunxi. Management problems in the construction of small water Conservancy projects [J]. Water Conservancy Technical Supervision, 3 (2018)

5. Zheng Jiarong, GONG Huili, LI Qingyuan, et al. Analysis of settlement control factors in Beijing Plain area from 2003 to 2009 based on PS-INSAR. Bulletin of Surveying and Mapping, 12 (2014)

6. Li Jiashan, Li Xiaowei. On the settlement monitoring technology of foundation subsidence area: Take the first Avenue of Qunli and rainwater pumping Station of Harbin as an example [J].Information on forestry science and technology, 51, 2 (2019)

7. Wang Lu, GUI Zhanfei. Application of GM-ARMABP Composite Model in building Settlement Prediction [J]. Beijing surveying and mapping, 33, 9 (2019)

8. Feng Shaoquan, Hua Xianghong, Tao Wuyong, et al. A Prediction model of pier settlement for high-speed Railway Based on GA-BP-MC Neural Network [J].Bulletin of Surveying and Mapping, (2019)

9. Song Chuping. An Improved BP Neural Network Method for deep Foundation Pit deformation prediction $[\mathrm{J}]$. Journal of civil engineering and management, 36, 5 (2019)

10. Li Wanglin, Tang Fuyu. Application of BP Neural Network in settlement Modeling of Composite Foundation [J]. Water Resources Planning and Design, 2 (2007) 